\title{
Anticancer Effect of Nemopilema nomurai Jellyfish Venom on HepG2 Cells and a Tumor Xenograft Animal Model
}

\author{
Hyunkyoung Lee, ${ }^{1}$ Seong Kyeong Bae, ${ }^{2}$ Munki Kim, ${ }^{3}$ Min Jung Pyo, ${ }^{2}$ \\ Minkyung Kim, ${ }^{2}$ Sujeoung Yang, ${ }^{2}$ Chung-kil Won, ${ }^{3}$ Won Duk Yoon, ${ }^{4}$ \\ Chang Hoon Han, ${ }^{4}$ Changkeun Kang, ${ }^{1,2}$ and Euikyung Kim ${ }^{1,2}$ \\ ${ }^{1}$ Institute of Animal Medicine, Gyeongsang National University, Jinju 660-701, Republic of Korea \\ ${ }^{2}$ College of Veterinary Medicine, Gyeongsang National University, Jinju 660-701, Republic of Korea \\ ${ }^{3}$ Department of Anatomy, College of Veterinary Medicine, Gyeongsang National University, Jinju 660-701, Republic of Korea \\ ${ }^{4}$ Headquarters for Marine Environment, National Fisheries Research \& Development Institute, Shiran-ri, Gijang-eup, \\ Gijang-gun, Busan 619-705, Republic of Korea
}

Correspondence should be addressed to Euikyung Kim; ekim@gnu.ac.kr

Received 2 February 2017; Accepted 5 June 2017; Published 13 July 2017

Academic Editor: Youn C. Kim

Copyright (c) 2017 Hyunkyoung Lee et al. This is an open access article distributed under the Creative Commons Attribution License, which permits unrestricted use, distribution, and reproduction in any medium, provided the original work is properly cited.

\begin{abstract}
Various kinds of animal venoms and their components have been widely studied for potential therapeutic applications. This study evaluated whether Nemopilema nomurai jellyfish venom (NnV) has anticancer activity. NnV strongly induced cytotoxicity of HepG2 cells through apoptotic cell death, as demonstrated by alterations of chromatic morphology, activation of procaspase-3, and an increase in the Bax/Bcl-2 ratio. Furthermore, NnV inhibited the phosphorylation of PI3K, PDK1, Akt, mTOR, p70S6K, and 4EBP1, whereas it enhanced the expression of p-PTEN. Interestingly, $\mathrm{NnV}$ also inactivated the negative feedback loops associated with Akt activation, as demonstrated by downregulation of Akt at Ser473 and mTOR at Ser2481. The anticancer effect of NnV was significant in a HepG2 xenograft mouse model, with no obvious toxicity. HepG2 cell death by NnV was inhibited by tetracycline, metalloprotease inhibitor, suggesting that metalloprotease component in $\mathrm{NnV}$ is closely related to the anticancer effects. This study demonstrates, for the first time, that $\mathrm{NnV}$ exerts highly selective cytotoxicity in HepG2 cells via dual inhibition of the Akt and mTOR signaling pathways, but not in normal cells.
\end{abstract}

\section{Introduction}

Hepatocellular carcinoma (HCC) is one of the most deadly cancers owing to its high rates of recurrence and metastasis [1]. HCC-related mortality is especially high in Asian and African countries. According to results published by GLOBOCAN 2012, the incidence of HCC in Korea is the sixth highest worldwide [2]. Despite advances in diagnostic imaging techniques and management of HCC, most patients with HCC are diagnosed with advanced-stage disease, which is regarded as incurable [3]. Chemotherapy for HCC is not satisfactory and the survival rate is extremely poor. Until now, the US Food and Drug Administration (FDA) has only approved one systemic treatment, sorafenib. Therefore, novel and more effective therapies for advanced HCC are necessary.
Venoms have attracted the attention of researchers engaged in identifying active components and developing novel drug candidates owing to their high sensitivity and specificity for target molecules. They have been used in traditional medicine, mainly in Asia and Africa. Cobra venom has been used to treat joint pain, inflammation, and arthritis in Indian traditional medicine, "Ayurveda" [4]. Bee venom has also been used to treat chronic inflammation (rheumatoid arthritis) and skin disease (acne and itch) and as pain relief for thousands of years $[5,6]$. Recently, drugs derived from venom have been developed and are being used for treating patients suffering from various diseases [7-10]. To date, there are six FDA-approved drugs derived from venoms and another ten drugs are being tested in clinical trials. 
Recently, jellyfish populations have dramatically increased owing to global warming and changes in the salinity of oceans. Jellyfish have nematocysts, which are specialized venom-containing cells in the tentacles. Under some stimuli or conditions involving skin contact, a nematocyst is rapidly discharged, which penetrates victims and delivers venom. Jellyfish venom is composed of various chemicals, including peptides, enzymes, neurotoxin, cytolysin, and hemolysin [11-17]. Jellyfish are regarded to be hazardous to other forms of life and cause waste in marine environments as they damage the fishery industry and cause health problems in humans $[18,19]$. However, recently, the potential bioactivity of jellyfish has been reported to exhibit insecticidal, immunostimulative, anticoagulant, and antihypertensive effects [20-23]. Active components isolated from Rhopilema esculentum jellyfish venom have been demonstrated to have insecticidal activities [23]. Aurelia aurita venom shows anticoagulant effects through strong fibrinogenolytic activity by cleaving the $A \alpha$ and $B \beta$ chains of fibrinogen molecules [24]. Therefore, jellyfish venom is an interesting and potentially useful resource. Nemopilema nomurai, one of the largest jellyfish species, can grow up to a diameter of $2 \mathrm{~m}$ and a weight of $200 \mathrm{~kg}$. They are widely distributed and bloom off the coasts of Korea, China, and Japan [19]. It has been reported that $N$. nomurai has antioxidant activity, so it is a potentially beneficial food for humans [20]. A collagen extract from $N$. nomurai can stimulate the production of immunoglobulin and cytokines without causing any allergic complications, indicating that it has a regulatory effect on the immune system [21]. Qniumucin, a glycoprotein derived from N. nomurai jellyfish, was found to inhibit degeneration of articular cartilage in an in vivo osteoarthritis model [22]. However, there is little information about the anticancer effect of Nemopilema nomurai venom. The present study explored the novel pharmacological effects of $\mathrm{NnV}$ on cancer cells, its anticancer activity, and its underlying molecular mechanisms of action.

\section{Materials and Methods}

2.1. Chemicals and Reagents. Bovine serum albumin (BSA), Dulbecco's Modified Eagle's Medium (DMEM), fetal bovine serum (FBS), penicillin, RPMI-1640, streptomycin, and trypsin were purchased from Gibco-BRL (Grand Island, NY, USA). Dimethyl sulfoxide (DMSO), 3-(4,5-dimethylthiazol2-yl)-2,5 diphenyltetrazolium bromide (MTT), and propidium iodide (PI) were from Sigma-Aldrich Inc. (St. Louis, MO, USA). Antibodies for caspase-7, caspase-9, caspase-3, BclxL Mcl-1, PARP, Bcl-2, p-Akt, p-PI3K, p-PDK, p-PTEN, pmTOR, p-p70S6K, p-S6K, p-4EBP1, p-eIF4E, and GAPDH were obtained from Cell Signaling Technology (Beverly, MA, USA). Terminal deoxynucleotidyl transferase dUTP nick end labeling (TUNEL) System Kit was procured from Calbiochem (Darmstadt, Germany). ALT, AST, and CK were obtained from IDEXX Laboratories Inc. (Westbrook, Maine USA). All other reagents used were of the purest grade available.
2.2. Jellyfish Collection and Preparation. The specimens of $N$. nomurai jellyfish were captured from the Korea Strait along the coasts of Geoje in September, 2012. Only tentacles were collected and transferred immediately to a laboratory for further preparation. Nematocysts were isolated from the dissected tentacles as described in a Bloom method with slight modification [25]. In brief, dissected tentacles were rinsed with cold seawater to remove debris. The tentacles were placed in three volumes of cold seawater for $24 \mathrm{~h}$ with gentle swirling for $30 \mathrm{~min}$ once daily at $4^{\circ} \mathrm{C}$. After autolysis, the supernatant was collected and centrifuged at $4,000 \mathrm{~g}$ for $10 \mathrm{~min}$ and the settled material was resuspended in fresh seawater and set for autolysis for $24 \mathrm{~h}$. This process was repeated for 3 days. The sediments were collected and centrifuged at $4,000 \mathrm{~g}$ for $10 \mathrm{~min}$ and washed several times with fresh sea water by centrifugation at $100 \mathrm{~g}$ at $4^{\circ} \mathrm{C}$ for $5 \mathrm{~min}$ until debris around nematocysts was almost removed. Finally, the undischarged nematocysts were collected, lyophilized, and stored at $-70^{\circ} \mathrm{C}$ until use.

2.3. Venom Extraction and Preparation. Venom was extracted from freeze-dried nematocysts using the technique described by Seymour et al. with a minor modification [26]. In brief, venom was extracted from $50 \mathrm{mg}$ of lyophilized nematocyst powder in $1 \mathrm{ml}$ of cold phosphate buffered saline (PBS, $\left.\mathrm{pH} 7.4,4^{\circ} \mathrm{C}\right)$ using glass beads $(0.5 \mathrm{~mm}$ in diameter). This mixture was shaken at $3000 \mathrm{rpm}$ for $30 \mathrm{~s}$, which was repeated for ten times with intermittent cooling on ice. The venom extracts were transferred to a new Eppendorf tube and centrifuged $(15,000 \mathrm{~g})$ at $4^{\circ} \mathrm{C}$ for $30 \mathrm{~min}$. The supernatant was used as NnV for the present study. Protein concentration of $\mathrm{NnV}$ was determined by Bradford (1976) method (Bio-Rad, CA, USA) [27].

2.4. Cell Culture. For finding the target cancer cells of $\mathrm{NnV}$, various cancer cell lines were used, including A549 (human pulmonary adenocarcinoma), HepG2 (human hepatocellular carcinoma), HT-29 (human colon adenocarcinoma), MCF7 (human breast carcinoma), MDA-MB-231 (human breast adenocarcinoma), HaCaT (keratinocyte), human dermal fibroblast (HDF), H9C2 (cardiomyocyte), and WB-F344 (liver epithelial cell) cells. The A549 cell was maintained in RPMI-1640 and HDF cell was cultured in $\mathrm{FGM}^{\mathrm{TM}}-2$ BulletKit $^{\mathrm{TM}}$ (Lonza Group Ltd., Basel, Switzerland) and the others were maintained in DMEM supplemented with $10 \%$ FBS and $100 \mu \mathrm{g} / \mathrm{ml}$ penicillin-streptomycin-amphotericin B solution. They were grown as monolayer cultures and kept at $37^{\circ} \mathrm{C}$ in a humidified atmosphere with $5 \% \mathrm{CO}_{2}$ for growth. WBF344 and HaCaT cells were kindly supplied by Professor Changkeun Kang (Gyeongsang National University, Jinju, Korea). HDF cells were purchased from MCTT (Modern cell \& Tissue Technologies, Inc., Seoul, Korea). Other cells used in the present study were purchased from American Type Culture Collection (ATCC).

2.5. Cell Viability. Cells were seeded in 24-well plates at a density of $4 \times 10^{4}$ cells/well. After $24 \mathrm{~h}$, the cells were treated for another $24 \mathrm{~h}$ with increasing concentrations of $\mathrm{NnV}$ 
$(0-10 \mu \mathrm{g} / \mathrm{ml})$. MTT solution $(5 \mathrm{mg} / \mathrm{ml})$ was then added to each well and incubated for additional $3 \mathrm{~h}$ at $37^{\circ} \mathrm{C}$. After the supernatant was removed, dimethyl sulfoxide (DMSO) was added to each well to dissolve the formazan crystal generated. Cell viability was determined by measuring optical density at $540 \mathrm{~nm}$ using ELISA multiwell plate reader (PowerWaveXS, BioTek Instruments, Inc., Winooski, USA).

2.6. Cell Cycle Analysis. Cells were seeded in 6-well plate at a density of $1 \times 10^{5}$ cells/well a day before experiment. After the treatment of $\mathrm{NnV}$ for $24 \mathrm{~h}$, both adherent and suspended cells were collected and pooled together for the analysis of cell cycle. The cell pellets were resuspended in $300 \mu \mathrm{L}$ of PBS and fixed by adding $700 \mu \mathrm{L} \mathrm{EtOH}$. The cells were incubated at $-20^{\circ} \mathrm{C}$ overnight and washed with fresh PBS again. The cellular DNA was stained with PI $(50 \mu \mathrm{g} / \mathrm{ml})$ in the presence of RNase A $(10 \mu \mathrm{g} / \mathrm{ml})$. A FACSCalibur flow cytometer (BD Biosciences, Franklin Lakes, NJ, USA) was used to analyze the cell cycle distribution.

2.7. Nuclear Morphology. For nuclear morphological analysis, HepG2 cells $\left(1 \times 10^{4}\right.$ cells/well) were plated onto cell culture slide (SPL life science, Pocheon, Korea) and treated with indicated concentrations of $\mathrm{NnV}(0-1.2 \mu \mathrm{g} / \mathrm{ml})$ for $24 \mathrm{~h}$. The cells on slide were fixed with $4 \%$ paraformaldehyde for $15 \mathrm{~min}$ and washed three times for $10 \mathrm{~min}$ each. Then they were stained with Hoechst 33342 fluorescent dye $(0.5 \mu \mathrm{g} / \mathrm{ml})$ for $15 \mathrm{~min}$. Nuclear morphology was observed by fluorescence microscopy (Leica, Wetzlar, Germany) using a 200x objective.

2.8. Western Blot Analysis. Cells were seeded in 6-well plate and were incubated with various concentrations of $\mathrm{NnV}$ for $24 \mathrm{~h}$. The treated cells were collected by scraping with $150 \mu \mathrm{L}$ of RIPA buffer containing protease inhibitor cocktail. Protein sample concentration was measured using the Bradford protein assay (Bio-Rad). Lysates $(50 \mu \mathrm{g})$ were separated by $12 \%$ SDS-polyacrylamide gel and then transferred into PVDF membranes (Bio-Rad, CA, USA). Western blots were probed with specific primary antibodies overnight at $4^{\circ} \mathrm{C}$. Following incubation with horseradish peroxidase-conjugated secondary antibody (Cell Signaling Technology, Beverly, MA) for $1 \mathrm{~h}$ at room temperature, the blots were visualized by using an enhanced chemiluminescence method (ECL, Amersham Biosciences, Buckinghamshire, UK) and analyzed using ChemiDoc XRS (Bio-Rad, CA, USA). Densitometry analysis was performed with a Hewlett-Packard scanner and $\mathrm{NIH}$ Image software (Image)).

2.9. Animals and Tumor Xenograft Model. Male athymic nude mice at the age of 4 weeks were purchased from the Central Lab Animal Incorporation (Seoul, Korea) and housed in the Animal Resource Facility at the Gyeongsang National University at Jinju in accordance with good animal practice as defined by the NIH guidelines. The animal protocol used in this study was approved by the Institutional Animal Care and Use Committee of the Gyeongsang National University at Jinju, and the animal protocol number is GNU-130129M0018.
HepG2 cells $\left(1 \times 10^{7}\right.$ in $200 \mu \mathrm{L}$ PBS $)$ were injected subcutaneously into the right flank of each mouse. When the tumor volume reached over $100 \mathrm{~mm}^{3}$, the animals were divided randomly into three groups with eight mice per group. The mice in control group were intraperitoneally (IP) injected with $200 \mu \mathrm{L}$ PBS once every other days for 20 days, while $\mathrm{NnV}$ treated groups were injected with either $\mathrm{NnV} 0.1$ or $0.3 \mathrm{mg} / \mathrm{kg}$. The tumor growth and body weight of each mouse were recorded once every other days during experimental period. Tumor sizes were measured using Vernier calipers and their volumes were calculated using the hemiellipsoidal model formula: tumor volumes $=$ [tumor length in $\mathrm{mm} \times$ (tumor width $^{2}$ in $\mathrm{mm}$ )]/2. The experiment was terminated 22 days after $\mathrm{NnV}$ treatment. At this time, mice were sacrificed, tumor from each mouse was excised, and wet weight of each tumor in each group was recorded. Each tumor tissue was fixed in $10 \%$ neutral buffered formalin and paraffinembedded and used for H\&E staining, TUNEL assay, and immunohistochemistry assay.

2.10. Biochemistry Analysis in Blood. Blood samples were collected from the caudal vena cava of the mice and then there was centrifugation at $3000 \times \mathrm{g}$ for $5 \mathrm{~min}$. These samples were used for determination of alanine transaminase (ALT), aspartate aminotransferase (AST), and creatinine kinase (CK) using clinical chemistry analyzer system (VetTest 8008; IDEXX Laboratories, Westbrook, ME, USA).

2.11. Histological Examination. Excised liver and heart from the control and $\mathrm{NnV}$-treated groups were fixed in $10 \%$ neutral buffered formalin and processed into paraffin blocks. Sections were deparaffinized in xylene and rehydrated in graded-alcohol washes. H\&E staining was performed using standard procedure. The specific staining was visualized and images were acquired using a light microscope with $200 x$ objectives (Olympus, Center Valley, PA, USA).

2.12. DNA Fragmentation Detection in Tumor Tissue. Apoptosis was detected using a terminal deoxynucleotidyl transferase dUTP nick end labeling (TUNEL) System Kit (Calbiochem). $5 \mu \mathrm{m}$ thick tumor tissue sections were deparaffinized and rehydrated and treated with $20 \mu \mathrm{g} / \mathrm{ml}$ proteinase $\mathrm{K}$ for $20 \mathrm{~min}$. The DNA was end-labeled with Biotinylated Nucleotide Mix in terminal deoxynucleotidyl transferase buffer at $37^{\circ} \mathrm{C}$ for $30 \mathrm{~min}$ and visualized by inverted fluorescence microscopy (Leica, Wetzlar, Germany). Total cell nuclei were stained by DAPI counterstaining. The quantification of TUNEL-positive cells was performed with a Hewlett-Packard scanner and NIH Image software (Image J).

2.13. Immunohistochemical Analysis in Tumor Xenografts. Immunohistochemistry (IHC) for Ki-67, p-Akt, and Ecadherin was performed using rabbit monoclonal antibodies. Tumor tissues were deparaffinized with xylene twice for $5 \mathrm{~min}$ and rehydrated and rinsed with tap water for $10 \mathrm{~min}$. They were incubated at $4^{\circ} \mathrm{C}$ with primary antibodies overnight. Immunoreactivity was visualized with an avidinbiotin peroxidase reaction (PK-4001, Vectastain ABC kit). 
The peroxidase reaction was developed using a 3,3'-diaminobenzidine tetrahydrochloride (D-5905, Sigma). The sections were counterstained with hematoxylin before being mounted.

2.14. Isolation of Bioactive Component with Anticancer Effect from $\mathrm{NnV}$. $\mathrm{NnV}$ was extracted in $10 \mathrm{Mm}$ Tris- $\mathrm{HCl}$ buffer $(\mathrm{pH}$ 7.8) and fractionated on DEAE cation-exchange column. The proteins were eluted with a linear gradient of $\mathrm{NaCl}(0-80 \%)$ using three column volumes of eluting phosphate buffer. The flow rate was adjusted to $1 \mathrm{ml} / \mathrm{min}$ and $1.5 \mathrm{ml}$ fraction was collected in each tube. Each protein peak was checked for cytotoxicity on HepG2 cells. The fractions showing cytotoxic activity were pooled and the protein amounts were measured.

2.15. Statistical Analysis. The results are expressed as mean \pm standard deviation (SD). One-way analysis of variance (ANOVA) was used to evaluate the significance of difference between two mean values. $p<0.05$ was considered to be statistically significant.

\section{Results}

3.1. NnV Is Selectively Cytotoxic toward HepG2 Cells. To determine the potential anticancer effect of $\mathrm{NnV}$ on various cancer cells, A549, HepG2, HT-29, MCF-7, and MDAMB231 cells were treated with $\mathrm{NnV}$ for $24 \mathrm{~h}$, and then an MTT assay was performed. The proliferation of HepG2 and MDA-MB231 was markedly suppressed, with an $\mathrm{IC}_{50}$ of $1.27 \mu \mathrm{g} / \mathrm{ml}$ and $2.58 \mu \mathrm{g} / \mathrm{ml}$ in the two cell types, respectively, while there was little inhibitory effect on A549, HT-29, and MCF-7 cells (Figure 1(a)). Hence, HepG2 cells were chosen for further experiments examining the anticancer effect of $\mathrm{NnV}$. NnV inhibited the proliferation of HepG2 cells in a dose-dependent manner, while showing no toxicity in normal cells at the same concentrations (Figure 1(b) and Supplementary Figure 1, in Supplementary Material available online at https://doi.org/10.1155/2017/2752716). To confirm whether the antiproliferative effect of $\mathrm{NnV}$ is associated with apoptosis, cell cycle analysis using flow cytometry was carried out. The subG1 population (indicating induction of apoptosis) in HepG2 cells was increased after treatment with $\mathrm{NnV}$ in a dose-dependent manner from 3.05\% to $69.44 \%$ (Figure 1(c)). In addition, NnV-treated HepG2 cells were observed to undergo nuclear fragmentation and cytoplasmic shrinkage via Hoechst staining (Figure $1(\mathrm{~d})$ ). These results suggest that $\mathrm{NnV}$ strongly inhibits the growth of HepG2 cells through induction of apoptotic cell death, but not that of normal cells.

\subsection{NnV Regulates Caspase Activation and Pro/Antiapoptotic} Protein Expression in HepG2 Cells. The apoptotic process is mainly executed by caspases. Caspase- 9 is the essential initiator and caspase- 3 and caspase- 7 are the effectors required for mitochondrial apoptosis [28]. To explore the molecular mechanisms of action of $\mathrm{NnV}$ on apoptotic HepG2 cell death, we measured the expressions of key caspase proteins. As shown in Figure 2(a), the expressions of procaspase-3, procaspase- 7 , and procaspase- 9 were dramatically decreased, while cleaved caspase-3, caspase-7, and caspase- 9 were increased by $\mathrm{NnV}$ treatment in a dose-dependent manner. Furthermore, it is well known that cleavage of poly-ADP ribose polymerase (PARP) by activation of caspase-3 plays a critical role in apoptosis through blocking DNA repair [29]. As expected, the expression of full-length PARP was decreased and that of cleaved PARP was increased in NnVtreated HepG2 cells. The expression levels of Bcl-xL, Bcl2 , and $\mathrm{Mcl}-1$ were also significantly reduced in $\mathrm{NnV}$-treated HepG2 cells, whereas the level of Bax was considerably elevated (Figure 2(b)). These results indicate that $\mathrm{NnV}$ induces apoptotic cell death in HepG2 cells.

\subsection{NnV Inhibits the PI3K/Akt/mTOR Signaling Pathway} in HepG2 Cells. The PI3K/Akt/mTOR signaling pathway is overexpressed or activated in various tumors and may inhibit apoptotic cell death and enhance survival and resistance to chemotherapy of cancer cells $[30,31]$. To elucidate whether $\mathrm{NnV}$-induced apoptosis is associated with the PI3K/Akt/ mTOR signaling pathway, alterations in the expression of its signaling proteins were detected. In HepG2 cells, NnV treatment decreased the levels of p-PI3K, p-PDK1 (Ser241), and p-Akt (Thr308), whereas it increased the phosphorylation of phosphatase and tensin homolog (PTEN), a negative regulator of the PI3K/Akt pathway (Figure 3(a)). Additionally, $\mathrm{NnV}$ decreased the expression of p-mTOR (Ser2448), pS6K1 (Thr389), and p-4E-BP1 (Thr37/46) (Figure 3(b)). NnV also suppressed p-S6 (downstream of S6K1) and p-eIF4E (a substrate of 4E-BP1) in a dose-dependent manner. PI3K/Aktmediated mTOR1 inhibition promotes negative feedback loops, which lead to cancer cell proliferation, migration, and survival through activation of Akt at Ser473 [32, 33]. Interestingly, NnV inhibited phosphorylation of Akt at Ser473 and p-mTOR at Ser2481 (indicator of mTOR2 activation) (Figures 3(a) and 3(b)). To confirm whether PI3K/Akt/mTOR signaling is necessary for the induction of apoptosis by $\mathrm{NnV}$, HepG2 cells were pretreated with LY294002 (an Akt inhibitor). Cotreatment with LY294002 and NnV significantly increased the expressions of procaspase-3, PARP, and $\mathrm{Bcl}-2$, indicating that the anticancer effect of $\mathrm{NnV}$ is closely associated with inactivation of the PI3K/Akt/mTOR signaling pathway (Figure 3(c)).

3.4. NnV Inhibits Hepatic Tumor Growth In Vivo. The in vitro study demonstrated that $\mathrm{NnV}$ has an anticancer effect on HepG2 cells through dual inhibition of Akt and mTOR. Therefore, the impact of $\mathrm{NnV}$ on hepatic tumor growth was assessed using a xenograft mouse model. After tumor volume increased to over $100 \mathrm{~mm}^{3}$, the tumor-bearing mice were treated with $\mathrm{NnV}(0.1$ and $0.3 \mathrm{mg} / \mathrm{kg})$ once every two days for 20 days. Tumor volume in the control group increased throughout the experimental period, whereas tumor volume in NnV-treated mice significantly decreased from the start of treatment until the end of the experiment (Figure 4(a)). On the 22 nd day, the tumor volume reduction rates were $81.9 \%$ and $82.8 \%$ in the mice treated with $\mathrm{NnV}$ at doses of 0.1 and $0.3 \mathrm{mg} / \mathrm{kg}$, respectively. Tumor growth in NnV-treated mice was also dramatically reduced (Figure 4(b)). During the 


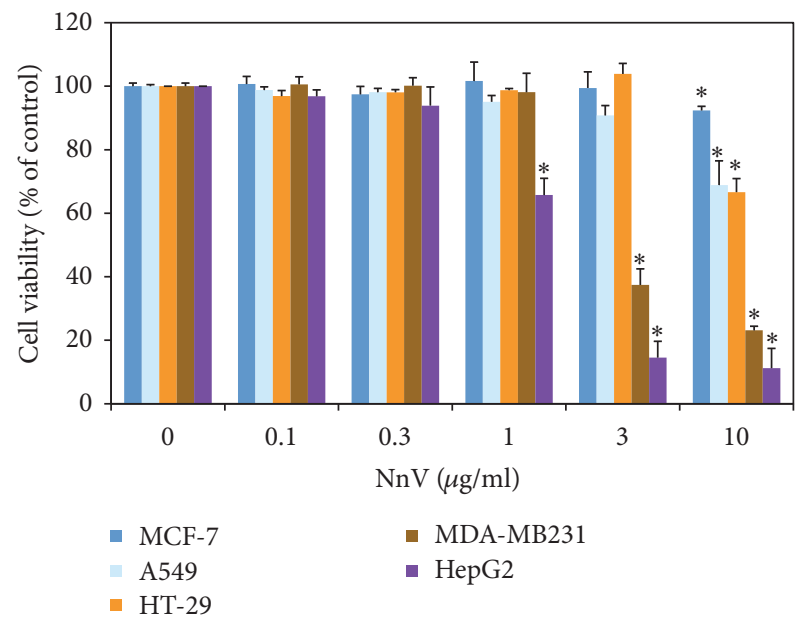

(a)
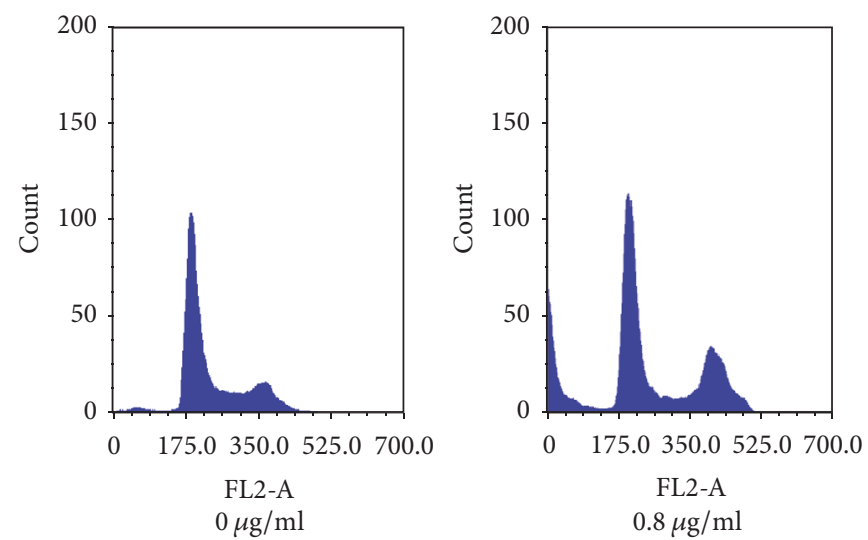

(c)

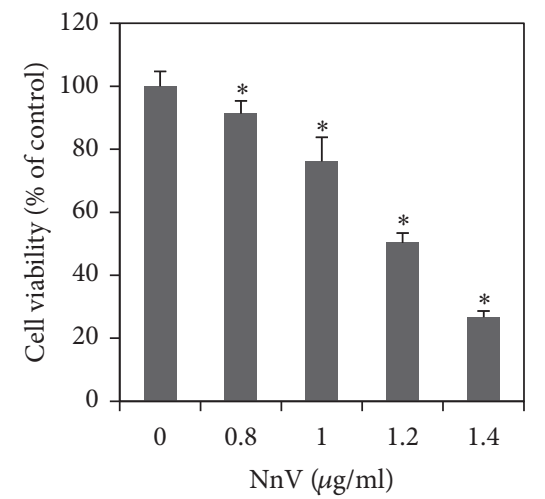

(b)
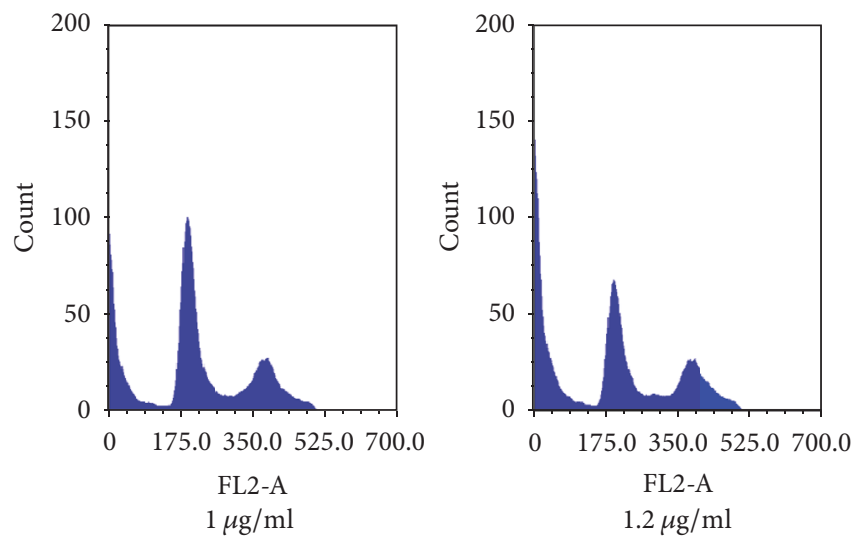
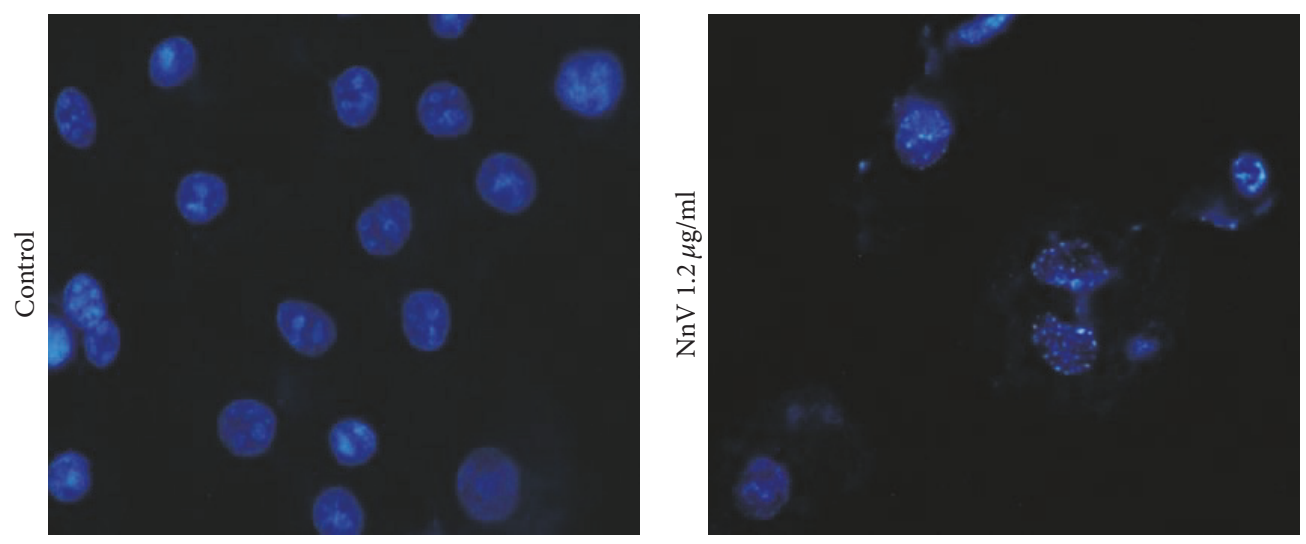

(d)

Figure 1: Effects of NnV on the cell viability in different cancer cells. (a) Cancer Cells (A549, HT-29, MCF-7, MDA-MB231, and HepG2 cells) were incubated for $24 \mathrm{~h}$ at various concentrations $(0,0.3,1,3$, and $10 \mu \mathrm{g} / \mathrm{ml})$ of NnV. Cell viability was analyzed by the MTT assay. (b) To determine efficient concentrations of cytotoxicity in HepG2, the cells were incubated for $24 \mathrm{~h}$ with various concentrations $(0-1.4 \mu \mathrm{g} / \mathrm{ml})$. (c) Apoptosis was measured by PI staining followed by flow cytometry analysis. (d) Hoechst 33342 fluorescence dye staining was performed and nuclear morphology was examined by fluorescence microscopy (Leica) using a 400x objective. The data shown are the mean \pm SD of six independent experiments. ${ }^{*} p<0.05$ was considered to indicate statistical significance compared with nontreated controls. 


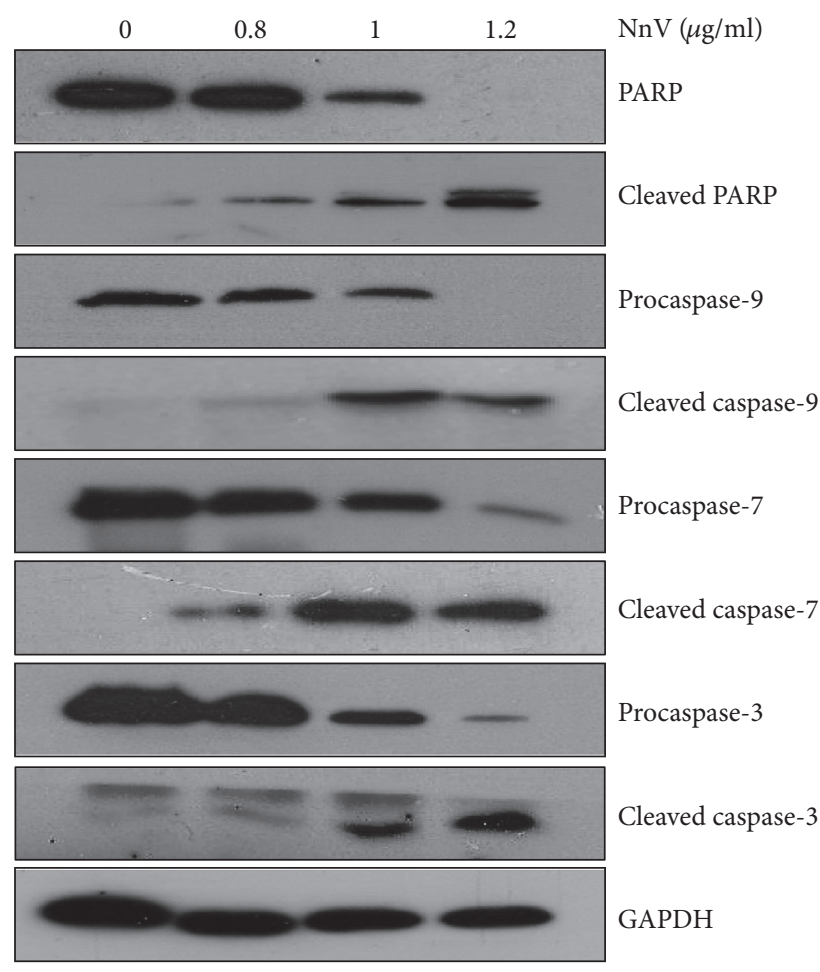

(a)

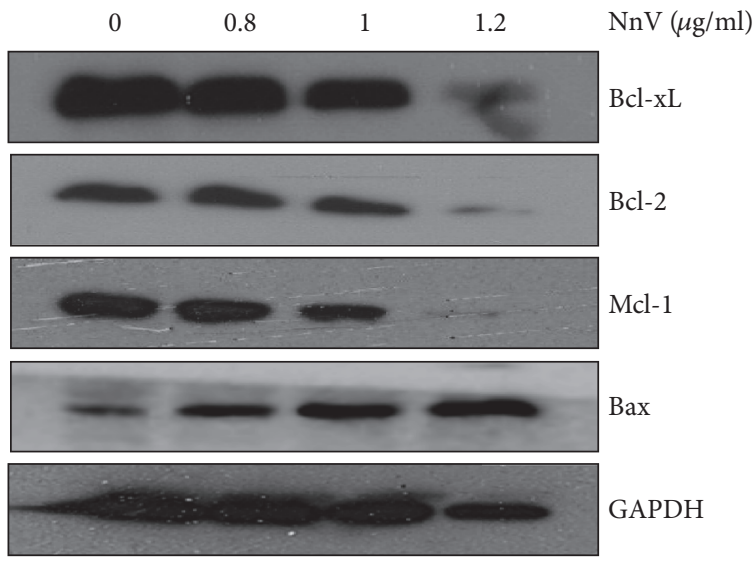

(b)

FIGURE 2: Effects of NnV on Bcl-2 family proteins and caspases in HepG2 cells. (a) HepG2 cells were treated with NnV (0, 0.8, 1, and 1.2 $\mu \mathrm{g} / \mathrm{ml})$. The levels of apoptosis-related proteins were analyzed by western blotting using specific monoclonal antibodies. (b) The expressions of Bcl-2 family proteins were analyzed by western blotting using specific monoclonal antibodies. GAPDH was used as a loading control.

experimental period, we observed no death or side effects in all experimental mice. Although the levels of AST, ALT, and $\mathrm{CK}$ were increased in $\mathrm{NnV}$-treated groups, their level was normal. Furthermore, there were no significant changes in body weight and histological alteration in heart or liver tissue in NnV-treated mice, indicating that effective doses of $\mathrm{NnV}$ are relatively safe (Supplement Figure 2).

\subsection{NnV Induces Apoptosis Partly through Akt and mTOR Sig-} naling in the Xenograft Model. To confirm the mechanism of the anticancer effect exerted by $\mathrm{NnV}$ in xenograft tissues, we performed the TUNEL and IHC assays. TUNEL-positive cells were more prevalent in NnV-treated tumor tissue in a dosedependent manner, and their numbers were significantly greater, by $4.5-$ and 7.8 -fold, respectively, in the $\mathrm{NnV} 0.1$ and $0.3 \mathrm{mg} / \mathrm{kg}$ groups (Figure $4(\mathrm{c})$ ). The IHC assay demonstrated that the expression of $\mathrm{Ki}-67$ was remarkably reduced in NnV-treated tumor tissue (Figure 4(b)). The expression of p-Akt at Thr308 and Ser473 was also diminished in NnVtreated tumor tissue. Interestingly, $\mathrm{NnV}$ enhanced $\mathrm{E}$-cadherin expression, indicating that $\mathrm{NnV}$ may affect specialized cellcell connections and cell-cell matrix interactions.

3.6. The Anticancer Effect of NnV Is Mainly Associated with Metalloprotease. To confirm the bioactive component of the anticancer effect of $\mathrm{NnV}$, we performed a cytotoxicity test using tetracycline (a metalloprotease inhibitor, Tetra). NnV induced 50\% cytotoxicity, whereas treatment with Tetra produced an almost full recovery of cell viability, indicating that the anticancer effect of $\mathrm{NnV}$ is closely associated with metalloprotease (Figure 5(a)). Hence, we tried to isolate the bioactive component using a DEAE ion exchange column. We observed three peaks, each of which was assessed by MTT assay (Figure 5(b)). That showed different results in that the viability of HepG2 cells was inhibited strongly by NnV-F1 and NnV-F2, but not NnV-F3 (Figure 5(c)). NnV-F1 and NnVF2 induced cytotoxicity at levels comparable to that of crude venom $(\mathrm{NnV})$, but $\mathrm{F} 3$ induced less cytotoxicity.

\section{Discussion}

There have been tremendous efforts by many investigators to discover novel components derived from the venom of various animals and to elucidate their modes of action, with the aim of developing therapeutic medicines, especially for the treatment of cancer. Jellyfish is a very interesting source owing to the high sensitivity and selectivity of its venom. As jellyfish blooms have increased in various parts of the world, there has been increased interest in the beneficial use of jellyfish venom, including its antiarthritic, antihypertensive, immunostimulatory, and insecticidal effects [21-23, 34, 35]. However, little is known about the beneficial effects of NnV. The present study investigated the anticancer therapeutic potential and mechanisms of action of $\mathrm{NnV}$ for the first time. 


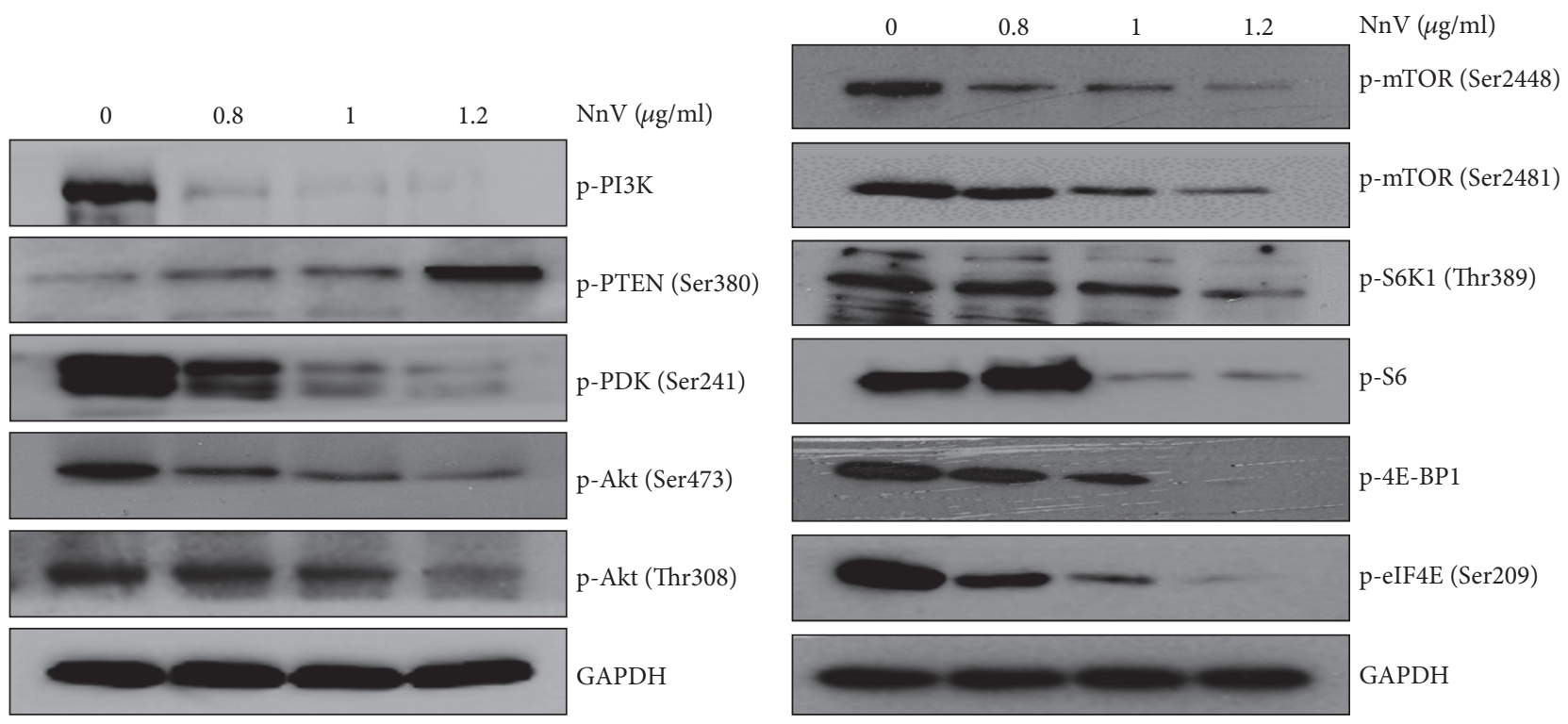

(a)

(b)

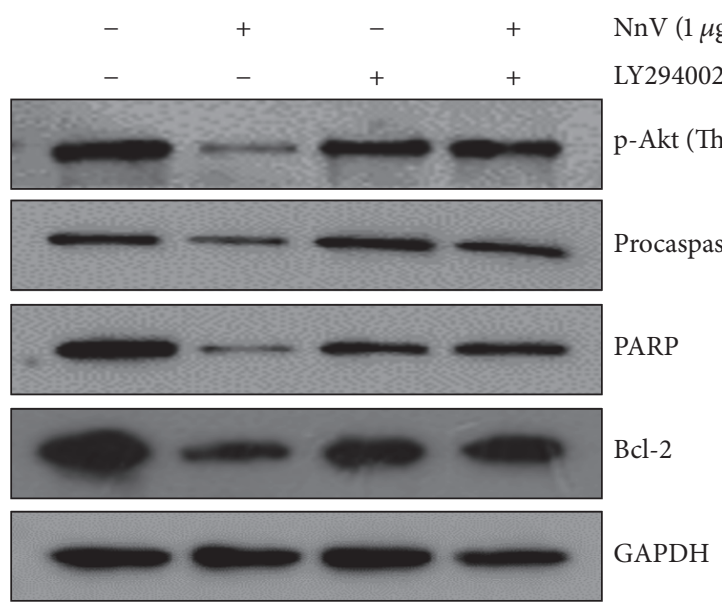

(c)

Figure 3: Effects of NnV on PI3K/Akt pathway in HepG2 cells. (a) The levels of p-PI3K, p-PTEN, p-PDK1, and p-Akt were analyzed by western blotting using specific monoclonal antibodies. GAPDH was used as a loading control. (b) The levels of p-mTOR, p-p70S6K, p-S6K, p-4E-BP1, and p-eIF4E were analyzed by western blotting using specific monoclonal antibodies. (c) HepG2 cells were pretreated with LY294002 (20 $\mu \mathrm{M})$ for $1 \mathrm{~h}$ and then treated with $\mathrm{NnV}$.

Various animal venoms have anticancer effects as demonstrated by inhibition of cell proliferation, migration, invasion, apoptosis, and angiogenesis. According to reports on anticancer effect of bee venom, the $\mathrm{IC}_{50}$ values were $14.2,6.3$, and $6.1 \mu \mathrm{g} / \mathrm{ml}$ in LNCaP, DU145, and PC-3 cells, respectively, after $72 \mathrm{~h}$ of treatment [36]. Another group reported that bee venom inhibited lung cancer cell growth at doses of 2.91 and $3.14 \mu \mathrm{g} / \mathrm{ml}$ in A549 and NCI-H460 cells, respectively [37]. Furthermore, an effective dose of bufalin (derived from toad skin and parotid venom) in pancreatic cancer cells was $0.1 \mu \mathrm{M}$ $(=3.865 \mu \mathrm{g} / \mathrm{ml})$, and ruviprase, a peptide purified from Russell's viper venom, induced apoptotic MCF-7 breast cancer cell death at $4 \mu \mathrm{g} / \mathrm{ml}[38,39]$. The present findings showed that NnV selectively inhibits the proliferation of HepG2 cells at doses of $0.8-1.2 \mu \mathrm{g} / \mathrm{ml}$ through induction of apoptotic cell death. Additionally, it is not toxic to normal hepatic epithelial cells, fibroblasts, and keratinocytes at the same concentrations. We did not observe any toxic or adverse effects in liver and heart tissues, such as changes in the level of functionrelated enzymes, findings on gross autopsy, or histopathologic changes in vivo. Therefore, the anticancer activity of $\mathrm{NnV}$ is as competitive as that of any other venoms.

To understand how NnV induces apoptosis, we investigated the PI3K/Akt/mTOR signaling pathway. The PI3K/Akt/ mTOR signaling pathway is one of the major pathways that regulates cell proliferation, migration, angiogenesis, and survival [40]. This pathway is thought to be the most frequently aberrant pathway in numerous cancers, affecting some $30-50 \%$ of tumors, making it an attractive target for anticancer drugs [41]. Pathological activation of its signaling can 


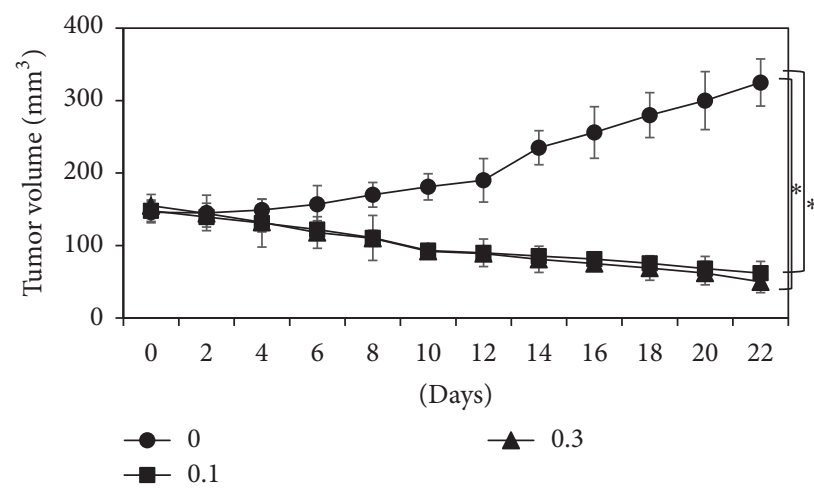

(a)
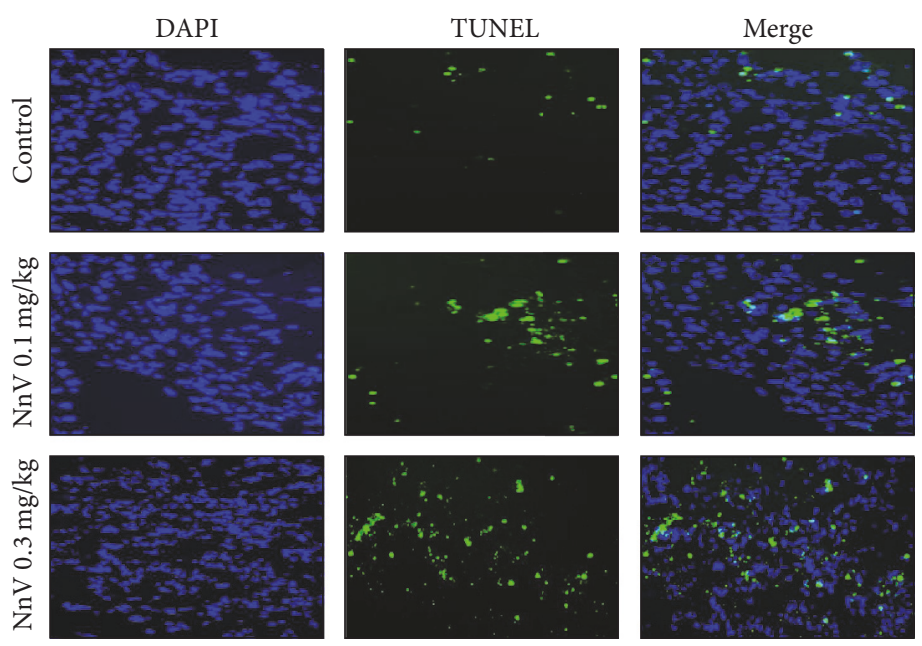

(c)

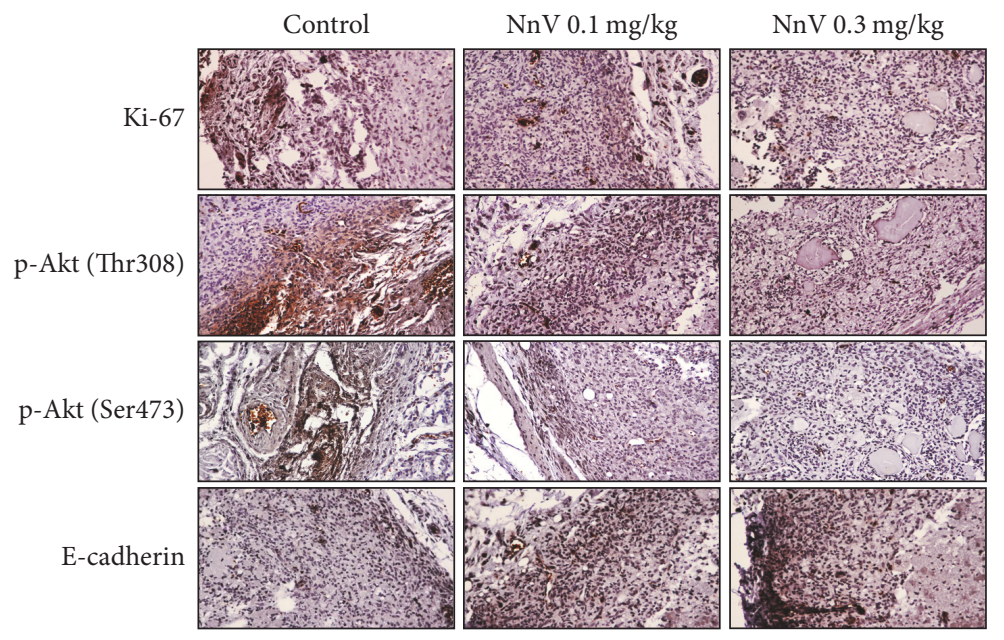

(d)

(b)
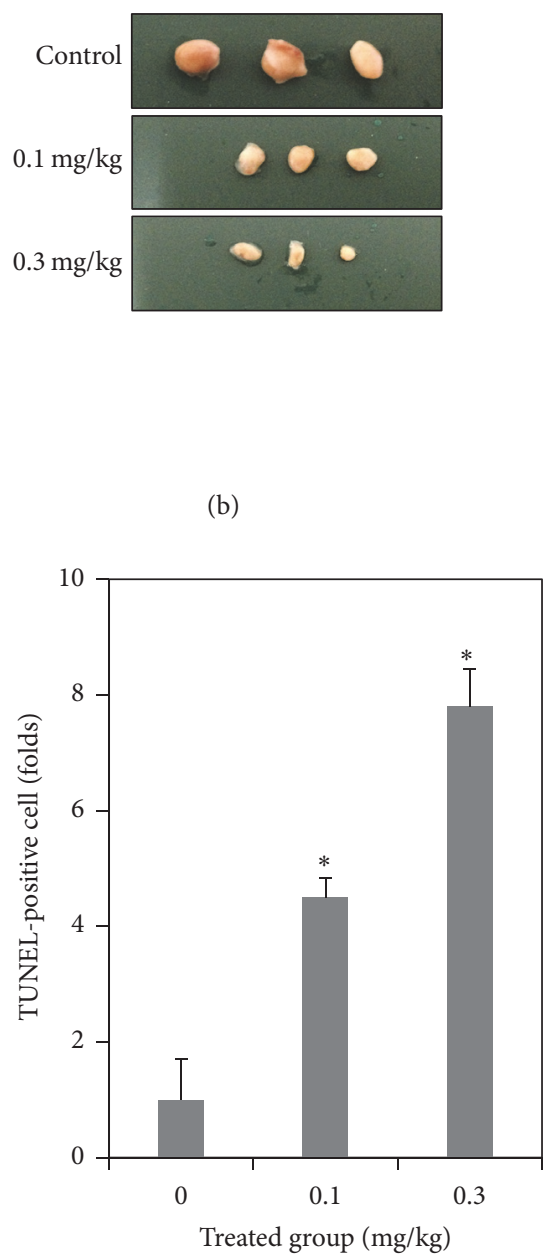

(1)

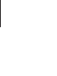

FIGURE 4: Effects of NnV on HepG2 tumor growth and induction of apoptosis. (a) Mice were orthotopically implanted with HepG2 cells and treated with $\mathrm{NnV}(0.1$ and $0.3 \mathrm{mg} / \mathrm{kg}$, I.P) for 20 day as described in Materials and Methods. Tumor sizes were measured by microcalipers every two days and average tumor volumes were representing the total tumor volumes per mouse. (b) On day 22, mice were sacrificed and the tumors were collected. Representative photographs of tumors are shown. (c) Induction of apoptosis was analyzed by TUNEL assay. As shown in the right panel, number of TUNEL-positive cells presented as folds compared with control group. (d) Tumor tissues were analyzed by immunohistochemistry to identify Ki-67 and p-Akt at Ser 473 and Thr308 and E-cadherin expressions ( $\times 200$ objective magnification). The results are expressed as the mean $\pm \mathrm{SD} .{ }^{*} p<0.01$ was considered to indicate statistical significance compared with control group. 


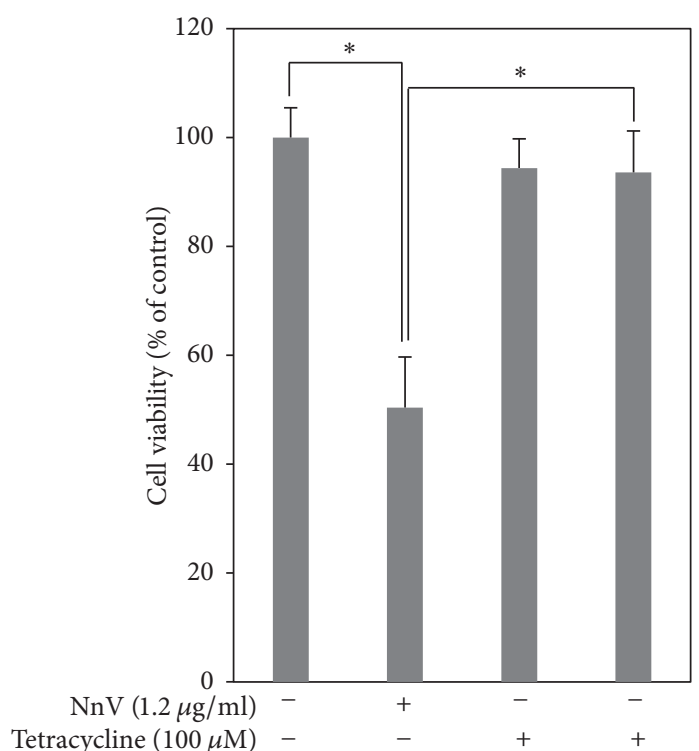

(a)

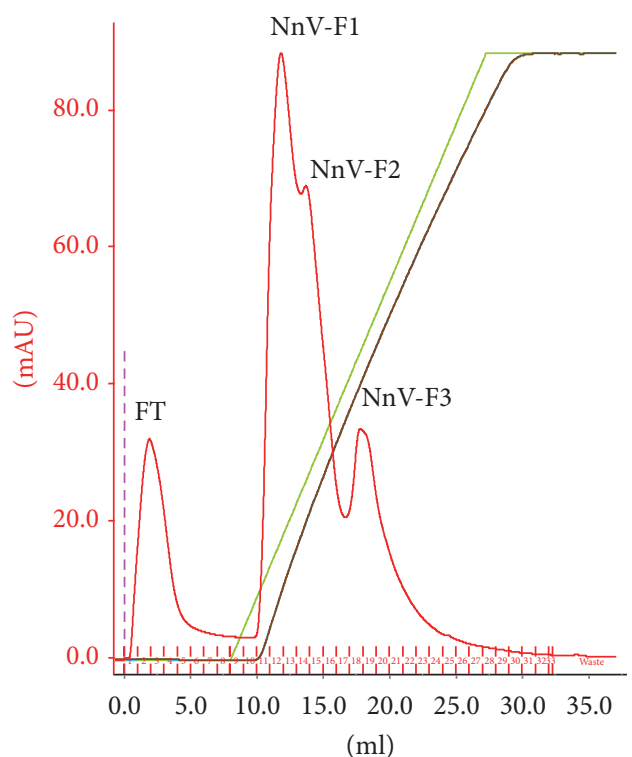

(b)

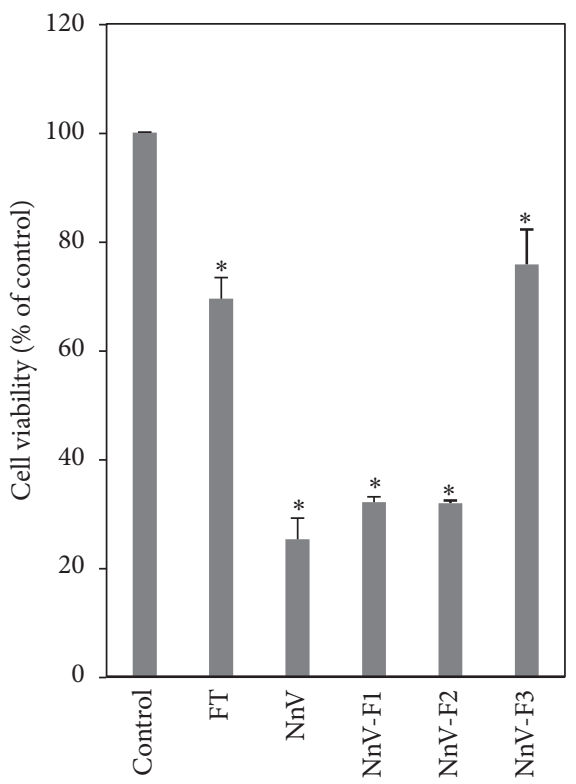

(c)

Figure 5: Isolation of anticancer component from NnV. (a) NnV preincubated with tetracycline (Tetra) $150 \mu \mathrm{M}$ for $3 \mathrm{~h}$ was treated in HepG2 cells for $24 \mathrm{~h}$ and then MTT assay was performed. (b) Crude venom was dissolved in $10 \mathrm{mM}$ Tris- $\mathrm{HCl}$ (pH 7.8) buffer and centrifuged for $30 \mathrm{~min}$ at $13,000 \times \mathrm{g}$. The supernatant was introduced in a DEAE column and the proteins were eluted with a $\mathrm{NaCl}$ linear gradient of 0 to $80 \%$ at flow rate of $1 \mathrm{ml} / \mathrm{min}$. (c) All fractions were evaluated for cytotoxicity in HepG2 cells.

occur in multiple ways, with the most common being point mutations in PI3K, loss of PTEN function, and hyperactivation of Akt. Akt is overexpressed in up to to $71.5 \%$ of HCC samples, leading to invasion, metastasis, and vascularization of HCC [40]. Aberrant mTOR signaling has been detected in up to $47.5 \%$ of HCC cases and results in poor prognosis [42]. Therefore, the PI3K/AKT/mTOR signaling pathway is a suitable therapeutic target in HCC. NnV dramatically decreased phosphorylation of PI3K, PDK1, and Akt at Thr308, whereas it significantly upregulated the expression of PTEN. Furthermore, NnV inhibited the phosphorylation of mTOR, which is downstream of Akt. NnV suppressed the phosphorylation of S6K1 and 4E-BP1, two well-characterized direct substrates of mTOR1, in a dose-dependent manner. Consistently, $\mathrm{NnV}$ suppressed phosphorylation of S6 and eIF4E. Despite the inactivation of PI3K-mediated p-Akt (Thr308), mTOR1 inhibition still promotes feedback activation of mTOR2-driven phosphorylation of Akt at Ser473 [32, 33]. Inhibition of 
PI3K/Akt-mediated mTOR signaling affects mTOR1, but not mTOR2. A compensatory feedback loop results in activation of mTOR2-driven Akt phosphorylated at Ser473. Akt is both an upstream activator of mTOR1 and a downstream effector of mTOR2 $[32,42]$. Therefore, dual inhibition of PI3K/Akt and mTOR is an effective strategy for targeting cancer cells. We examined whether $\mathrm{NnV}$ inhibited mTOR2 signaling. $\mathrm{NnV}$ repressed p-Akt at Ser473 (an mTOR2 target site) as well as p-mTOR at Ser2481 (an indicator of mTOR2 activation) in HepG2 cells. Additionally, inactivation of Akt subsequently induces the mitochondrial apoptotic pathway through alterations in the ratio of Bax to Bcl-2 and activation of caspase3. The caspase cascade is a vital hallmark of apoptosis which results in regulation of pro-/antiapoptotic proteins (Bax, Bcl2, $\mathrm{Bcl}-\mathrm{xL}$, and $\mathrm{Mcl}-1)$. The $\mathrm{Bax} / \mathrm{Bcl}-2$ ratio is critical for the induction of apoptosis and determines whether or not cells will undergo apoptosis [43]. NnV treatment resulted in decreases in the levels of the antiapoptotic proteins $\mathrm{Bcl}-2$, $\mathrm{Bcl}-\mathrm{xL}$, and Mcl-1 but led to an increase in the level of the proapoptotic protein Bax. The Bax/Bcl-2 ratio was dramatically increased from 1 to 134 by $\mathrm{NnV}$ treatment, leading to activation of caspase-3, caspase-7, and caspase- 9 and inactivation of PARP. These results indicate that $\mathrm{NnV}$ promotes apoptosis through increasing the $\mathrm{Bax} / \mathrm{Bcl}-2$ ratio and subsequent activation of caspases.

We further conducted an in vivo study using xenograft animal models. As expected, NnV strongly suppressed tumor growth in HepG2-bearing mice through induction of apoptotic HepG2 cell death. Compared with the control group, tumor volume in the $\mathrm{NnV}$-treated groups decreased by nearly $80 \%$. The NnV-treated groups showed a remarkable reduction in the expression of Ki-67- (indicators of cellular proliferation) and an increase in the number of TUNEL-positive cells, indicating that $\mathrm{NnV}$ induces inhibition of cancer cell proliferation and apoptotic cell death. Based on our in vitro study, the effects of $\mathrm{NnV}$ on the Akt pathway were investigated in a HepG2 xenograft model. NnV exhibited dual inhibition of Akt at Thr 308 and Ser 473, which further suggests that $\mathrm{NnV}$ displays anticancer activity related to suppression of both Akt and mTOR. Interestingly, it was observed that $\mathrm{NnV}$ treated tumor tissues are less diffused and more compact compared with tissues in the control group. To confirm whether $\mathrm{NnV}$ affects cell-cell density and cell-cell matrix interactions in tumor tissue, we assessed the expression of E-cadherin, which is an important protein involved in cell adhesion. The loss of its function promotes cellular motility and invasiveness by decreasing the strength of cellular adhesion, contributing to cancer cell progression and metastasis. $\mathrm{NnV}$ dose-dependently increased expression of E-cadherin in the HepG2 xenograft model, indicating that $\mathrm{NnV}$ may inhibit the migration and invasion of HepG2 cells by regulating E-cadherin. Further studies are necessary to demonstrate whether the novel anticancer effect of $\mathrm{NnV}$ is associated with regulation of the epithelial-to-mesenchymal transition (EMT).

Although this study demonstrates the anticancer effect of $\mathrm{NnV}$, identifying the molecular mechanisms associated with this activity is difficult because full identification and characterization of individual components of venom have not been done. A previous study and unpublished proteomic data (being prepared for publication) demonstrated that $\mathrm{NnV}$ contains an abundant amount of metalloprotease-like enzymes [16]. According to proteomic profile, 150 toxin proteins were identified by $2-\mathrm{DE}$, including metalloprotease, serine protease inhibitor, phospholipase, and serine protease. Metalloproteases were major component in the N. nomurai venom with a proportion of $21 \%$ of the identified venom toxins. In other venoms (snakes and spiders), metalloprotease exerted inhibitory effects on cancer cell adhesion, proliferation, migration, invasion, and angiogenesis. Several studies reported that venom metalloprotease has potential anticancer effects [44]. The extracellular matrix (ECM) plays important roles in cell behavior and function, including growth, death, adhesion, and migration. These changes are mainly involved with interaction of integrin receptors, which can lead to apoptosis in cancer cells. Anoikis, a special type of apoptosis, is provoked by degradation of ECM components such as matrix metalloproteinases (MMP) [45]. Jararhagin, a purified snake venom metalloprotease from Bothrops jararaca, exhibited an inhibitory effect on cell adhesion and a cytotoxic effect on melanoma cells $[46,47]$. TSV-DM, a metalloprotease from Trimeresurus stejnegeri, brought about inhibition of cell proliferation and induction of apoptotic cell death in ECV304 cells [48]. To investigate whether the anticancer effect of $\mathrm{NnV}$ is associated with metalloprotease, cell viability was assessed after preincubation with $\mathrm{NnV}$ and MMP inhibitors (tetracycline). $\mathrm{NnV}$ treatment alone caused $50 \%$ cytotoxicity in HepG2 cells, while cotreatment between $\mathrm{NnV}$ and Tetra had little effect on cell viability. Next, we tried to isolate the bioactive component that exerts the anticancer effect. We obtained three peaks, and each fraction was assessed for cytotoxicity in HepG2 cells. Compared with crude venom (NnV), F1 and F2 induced similar cytotoxicity, while NnV-F3 induced less cytotoxicity. To identify and characterize the active components that have an anticancer effect, sufficient amounts of NnVF1 and NnV-F2 are necessary. However, NnV-F1 and NnVF2 had very low protein concentrations, making it difficult to identify and characterize the active components. Other groups reported that identification of individual components of jellyfish venom is very difficult as a large amount of venom is necessary for isolation and characterization, and the venom has unstable properties and an insufficient amount of previously published data, unlike other types of venom (snake and scorpion) [17, 49]. Despite the potential of $\mathrm{NnV}$, obtaining sufficient amounts of venom for further studies for identification and characterization remains a significant challenge.

In conclusion, although purification of anticancer component from $\mathrm{NnV}$ has not succeeded, we propose new insights into possibility of $\mathrm{NnV}$ in pharmacological intervention of cancer therapy. In the next study, we will establish a series of purification steps for the isolation of anticancer component and perform more detailed studies on the mechanisms of its action. By any measure, this is the first report to demonstrate that $\mathrm{NnV}$ has a selective anticancer effect, which acts partly through dual inhibition of PI3K/Akt and mTOR signaling in HepG2 cells and a xenograft model. 


\section{Conflicts of Interest}

The authors declare that there are no conflicts of interest regarding the publication of this paper.

\section{Authors' Contributions}

Euikyung Kim conceived and designed the study. Hyunkyoung Lee and Seong Kyeong Bae mainly performed the experiments and wrote the manuscript. Min Jung Pyo, Minkyung Kim, and Sujeong Yang performed the in vivo study and analyzed the data. Munki Kim and Chung-kil Won performed histological experiments, including H\&E staining, IHC. Won Duk Yoon and Chang Hoon Han advised on jellyfish sampling and species identification. Changkeun Kang interpreted and discussed the data. All authors have read and approved the final manuscript. Hyunkyoung Lee and Seong Kyeong Bae contributed equally to this work.

\section{Acknowledgments}

This work was supported by the National Research Foundation of Korea (NRF) Grants by the Korea Government (NRF2015R1C1A1A02037588 and NRF-2014R1A2A2A01007245) and National Institute of Fisheries Science (R2017046 and 15-0E-14).

\section{References}

[1] A. Jemal, F. Bray, M. M. Center, J. Ferlay, E. Ward, and D. Forman, "Global cancer statistics," CA: A Cancer Journal for Clinicians, vol. 61, no. 2, pp. 69-90, 2011.

[2] J. Ferlay, I. Soerjomataram, R. Dikshit et al., "Cancer incidence and mortality worldwide: sources, methods and major patterns in GLOBOCAN 2012," International Journal of Cancer, 2014.

[3] D. Sloane, H. Chen, and C. Howell, "Racial disparity in primary hepatocellular carcinoma: tumor stage at presentation, surgical treatment and survival," Journal of the National Medical Association, vol. 98, no. 12, 1934.

[4] A. Gomes, S. Bhattacharya, M. Chakraborty, P. Bhattacharjee, and R. Mishra, "Anti-arthritic activity of Indian monocellate cobra (Naja kaouthia) venom on adjuvant induced arthritis," Toxicon, vol. 55, no. 2-3, pp. 670-673, 2010.

[5] Y.-H. Chang and M. L. Bliven, "Anti-arthritic effect of bee venom," Agents and Actions, vol. 9, no. 2, pp. 205-211, 1979.

[6] M. E. J. Billingham, J. Morley, J. M. Hanson, R. A. Shipolini, and C. A. Vernon, "An Anti-Inflammatory peptide from bee venom," Nature, vol. 245, no. 5421, pp. 163-164, 1973.

[7] J. Deshane, C. C. Garner, and H. Sontheimer, "Chlorotoxin inhibits glioma cell invasion via matrix metalloproteinase-2," Journal of Biological Chemistry, vol. 278, no. 6, pp. 4135-4144, 2003.

[8] G. F. King, "Venoms as a platform for human drugs: translating toxins into therapeutics," Expert Opinion on Biological Therapy, vol. 11, no. 11, pp. 1469-1484, 2011.

[9] R. Mir, S. Karim, M. A. Kamal, C. M. Wilson, and Z. Mirza, "Conotoxins: structure, therapeutic potential and pharmacological applications," Current Pharmaceutical Design, vol. 22, no. 5, pp. 582-589, 2016.
[10] G. Xu, D. A. Stoffers, J. F. Habener, and S. Bonner-Weir, "Exendin- 4 stimulates both $\beta$-cell replication and neogenesis, resulting in increased $\beta$-cell mass and improved glucose tolerance in diabetic rats," Diabetes, vol. 48, no. 12, pp. 2270-2276, 1999.

[11] Y. Ayed, A. Dellai, H. B. Mansour, H. Bacha, and S. Abid, "Analgesic and antibutyrylcholinestrasic activities of the venom prepared from the Mediterranean jellyfish Pelagia noctiluca (Forsskal, 1775)," Annals of Clinical Microbiology and Antimicrobials, vol. 11, article 15, 2012.

[12] H. Azuma, S. Sekizaki, A. Satoh, and T. Nakajima, "Platelet aggregation caused by carybdea rastonii toxins (CrTX-I, II, and III) obtained from a jellyfish, carybdea rastonii," Proceedings of the Society for Experimental Biology and Medicine, vol. 182, no. 1, pp. 34-42, 1986.

[13] S. Badré, "Bioactive toxins from stinging jellyfish," Toxicon, vol. 91, pp. 114-125, 2014.

[14] D. L. Brinkman and J. N. Burnell, "Biochemical and molecular characterisation of cubozoan protein toxins," Toxicon, vol. 54, no. 8, pp. 1162-1173, 2009.

[15] C. Kang, A. Munawir, M. Cha et al., "Cytotoxicity and hemolytic activity of jellyfish Nemopilema nomurai (Scyphozoa: Rhizostomeae) venom," Comparative Biochemistry and Physiology - C Toxicology and Pharmacology, vol. 150, no. 1, pp. 85-90, 2009.

[16] H. Lee, E.-S. Jung, C. Kang, W. D. Yoon, J.-S. Kim, and E. Kim, "Scyphozoan jellyfish venom metalloproteinases and their role in the cytotoxicity," Toxicon, vol. 58, no. 3, pp. 277-284, 2011.

[17] A. J. Weston, R. Chung, W. C. Dunlap et al., "Proteomic characterisation of toxins isolated from nematocysts of the South Atlantic jellyfish Olindias sambaquiensis," Toxicon, vol. 71, pp. 11-17, 2013.

[18] Z. Dong, D. Liu, and J. K. Keesing, "Jellyfish blooms in China: dominant species, causes and consequences," Marine Pollution Bulletin, vol. 60, no. 7, pp. 954-963, 2010.

[19] J. Fu, K. Koo, A. X. Sang, and D. C. Shisler, "Jellyfish envenomation in an ocean swimmer," Internal and Emergency Medicine, vol. 9, no. 1, pp. 103-104, 2014.

[20] B. Frazão and A. Antunes, "Jellyfish bioactive compounds: methods for wet-lab work," Marine Drugs, vol. 14, no. 4, article no. $75,2016$.

[21] X. Liu, M. Zhang, C. Zhang, and C. Liu, "Angiotensin converting enzyme (ACE) inhibitory, antihypertensive and antihyperlipidaemic activities of protein hydrolysates from Rhopilema esculentum," Food Chemistry, vol. 134, no. 4, pp. 2134-2140, 2012.

[22] S. Krishnan and P. Pachippan, "Imunomodulatory effects of the jelly fish venom C. quinquecirrha from vellar estuary, southeast coast of India," International Journal of Pharmacy and Pharmaceutical Sciences, vol. 5, no. 4, pp. 60-62, 2013.

[23] H. Yu, X. Liu, X. Dong et al., "Insecticidal activity of proteinous venom from tentacle of jellyfish Rhopilema esculentum Kishinouye," Bioorganic and Medicinal Chemistry Letters, vol. 15, no. 22, pp. 4949-4952, 2005.

[24] A. Rastogi, S. Biswas, A. Sarkar, and D. Chakrabarty, "Anticoagulant activity of Moon jellyfish (Aurelia aurita) tentacle extract," Toxicon, vol. 60, no. 5, pp. 719-723, 2012.

[25] D. A. Bloom, J. W. Burnett, and P. Alderslade, "Partial purification of box jellyfish (Chironex fleckeri) nematocyst venom isolated at the beachside," Toxicon, vol. 36, no. 8, pp. 1075-1085, 1998.

[26] J. Seymour, T. Carrette, P. Cullen, M. Little, R. F. Mulcahy, and P. L. Pereira, "The use of pressure immobilization bandages in 
the first aid management of cubozoan envenomings," Toxicon, vol. 40, no. 10, pp. 1503-1505, 2002.

[27] M. M. Bradford, "A rapid and sensitive method for the quantitation of microgram quantities of protein utilizing the principle of protein dye binding," Analytical Biochemistry, vol. 72, no. 1-2, pp. 248-254, 1976.

[28] R. Kim, M. Emi, and K. Tanabe, "Caspase-dependent and independent cell death pathways after DNA damage (Review)," Oncology Reports, 2005.

[29] A. H. Boulares, A. G. Yakovlev, V. Ivanova et al., "Role of poly(ADP-ribose) polymerase (PARP) cleavage in apoptosis. Caspase 3-resistant PARP mutant increases rates of apoptosis in transfected cells," The Journal of Biological Chemistry, vol. 274, no. 33, pp. 22932-22940, 1999.

[30] L. C. Cantley and B. G. Neel, "New insights into tumor suppression: PTEN suppresses tumor formation by restraining the phosphoinositide 3-kinase/AKT pathway," Proceedings of the National Academy of Sciences of the United States of America, vol. 96, no. 8, pp. 4240-4245, 1999.

[31] T. F. Franke, C. P. Hornik, L. Segev, G. A. Shostak, and C. Sugimoto, "PI3K/Akt and apoptosis: size matters," Oncogene, vol. 22, no. 56, pp. 8983-8998, 2003.

[32] H. A. Burris III, "Overcoming acquired resistance to anticancer therapy: focus on the PI3K/AKT/mTOR pathway," Cancer Chemotherapy and Pharmacology, vol. 71, no. 4, pp. 829-842, 2013.

[33] S. A. Wander, B. T. Hennessy, and J. M. Slingerland, "Nextgeneration mTOR inhibitors in clinical oncology: how pathway complexity informs therapeutic strategy," Journal of Clinical Investigation, vol. 121, no. 4, pp. 1231-1241, 2011.

[34] H. Morishige, T. Sugahara, S. Nishimoto et al., "Immunostimulatory effects of collagen from jellyfish in vivo," Cytotechnology, vol. 63, no. 5, pp. 481-492, 2011.

[35] N. Ohta, M. Sato, K. Ushida et al., "Jellyfish mucin may have potential disease-modifying effects on osteoarthritis," BMC Biotechnology, vol. 9, article no. 98, 2009.

[36] M. H. Park, M. S. Choi, D. H. Kwak et al., "Anti-cancer effect of bee venomin prostate cancer cells through activation of caspase pathway via inactivation of NF- $\kappa$ B," Prostate, vol. 71, no. 8, pp. 801-812, 2011.

[37] K. E. Choi, C. J. Hwang, S. M. Gu et al., "Cancer cell growth inhibitory effect of bee venom via increase of death receptor 3 expression and inactivation of NF-kappa B in NSCLC cells," Toxins, vol. 6, no. 8, pp. 2210-2228, 2014.

[38] X. Liu, X.-Y. Xiao, Q.-Y. Shou et al., "Bufalin inhibits pancreatic cancer by inducing cell cycle arrest via the c-Myc/NF- $\kappa \mathrm{B}$ pathway," Journal of Ethnopharmacology, vol. 193, pp. 538-545, 2016.

[39] R. Thakur, S. Kini, S. Kurkalang et al., "Mechanism of apoptosis induction in human breast cancer MCF-7 cell by Ruviprase, a small peptide from Daboia russelii russelii venom," ChemicoBiological Interactions, vol. 258, pp. 297-304, 2016.

[40] J.-S. Chen, Q. Wang, X.-H. Fu et al., "Involvement of PI3K/ PTEN/AKT/mTOR pathway in invasion and metastasis in hepatocellular carcinoma: association with MMP-9," Hepatology Research, vol. 39, no. 2, pp. 177-186, 2009.

[41] Y. Samuels, Z. Wang, A. Bardelli et al., "High frequency of mutations of the PIK3CA gene in human cancers," Science, vol. 304, no. 5670, p. 554, 2004.

[42] A. Villanueva, D. Y. Chiang, P. Newell et al., "Pivotal role of mTOR signaling in hepatocellular carcinoma," Gastroenterology, vol. 135, no. 6, pp. 1972.e11-1983.e11, 2008.
[43] D. Hanahan and R. A. Weinberg, "The hallmarks of cancer," Cell, vol. 100, no. 1, pp. 57-70, 2000.

[44] J. Chaisakul, W. C. Hodgson, S. Kuruppu, and N. Prasongsook, "Effects of animal venoms and toxins on hallmarks of cancer," Journal of Cancer, vol. 7, no. 11, pp. 1571-1578, 2016.

[45] I. Tanjoni, R. Weinlich, M. S. Della-Casa et al., "Jararhagin, a snake venom metalloproteinase, induces a specialized form of apoptosis (anoikis) selective to endothelial cells," Apoptosis, vol. 10, no. 4, pp. 851-861, 2005.

[46] M. C. Corrêa Jr., D. A. Maria, A. M. Moura-Da-Silva, K. F. Pizzocaro, and I. R. G. Ruiz, "Inhibition of melanoma cells tumorigenicity by the snake venom toxin jararhagin," Toxicon, vol. 40, no. 6, pp. 739-748, 2002.

[47] D. A. Maria, M. G. L. da Silva, M. C. Correia, and I. R. G. Ruiz, "Antiproliferative effect of the jararhagin toxin on B16F10 murine melanoma," BMC Complementary and Alternative Medicine, vol. 14, no. 1, article 446, 2014.

[48] S.-G. Wan, Y. Jin, W.-H. Lee, and Y. Zhang, "A snake venom metalloproteinase that inhibited cell proliferation and induced morphological changes of ECV304 cells," Toxicon, vol. 47, no. 4, pp. 480-489, 2006.

[49] A. A. Yanagihara and R. V. Shohet, "Cubozoan venom-induced cardiovascular collapse is caused by hyperkalemia and prevented by zinc gluconate in mice," PLOS ONE, vol. 7, no. 12, Article ID e51368, 2012. 


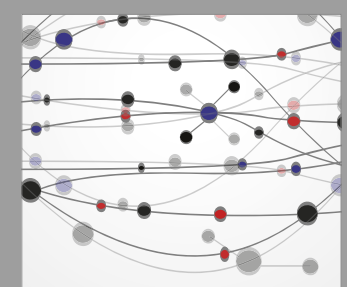

The Scientific World Journal
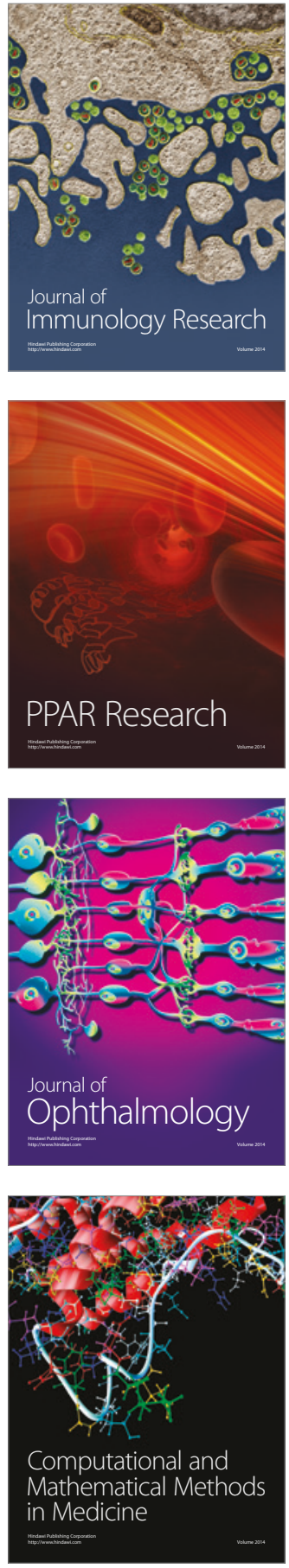

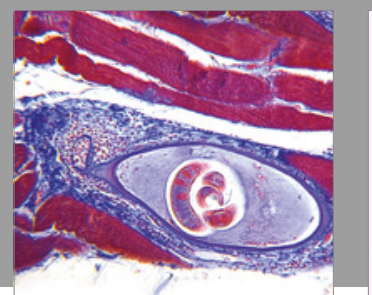

Gastroenterology Research and Practice
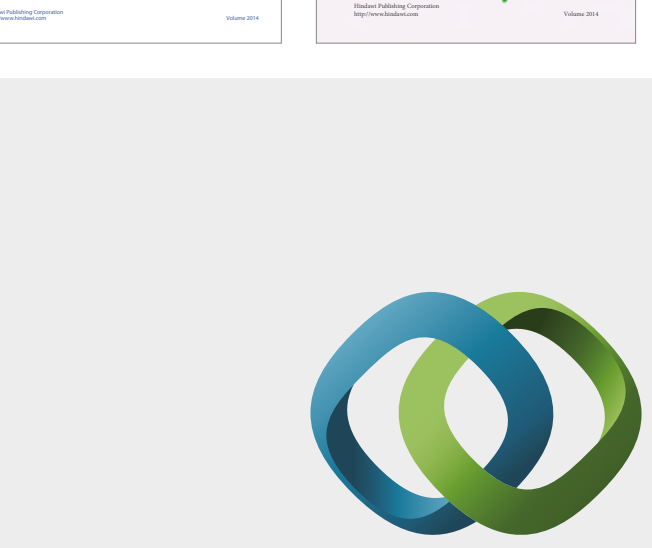

\section{Hindawi}

Submit your manuscripts at

https://www.hindawi.com
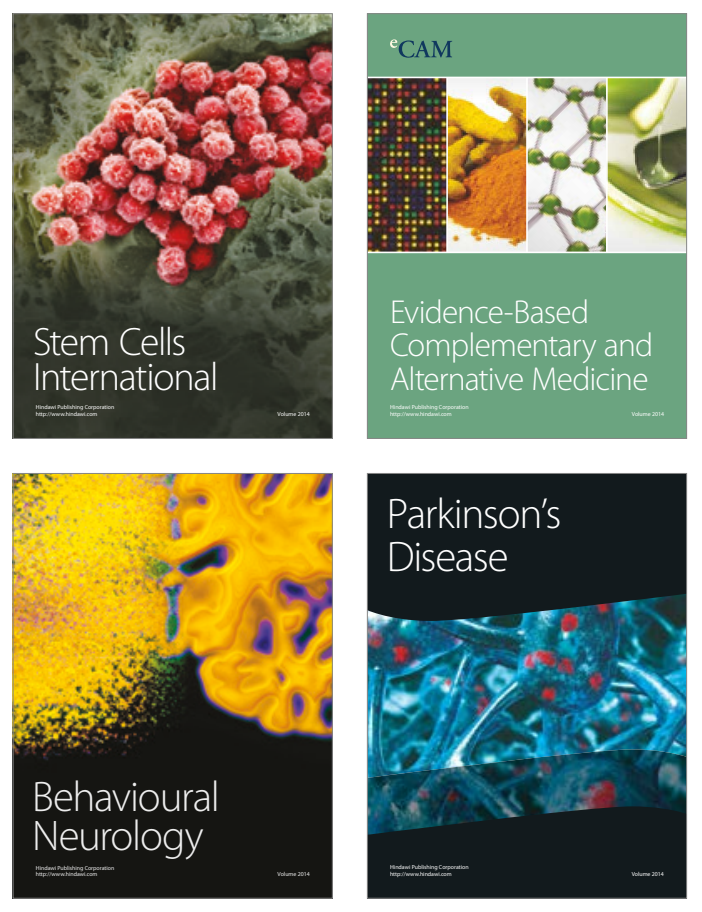
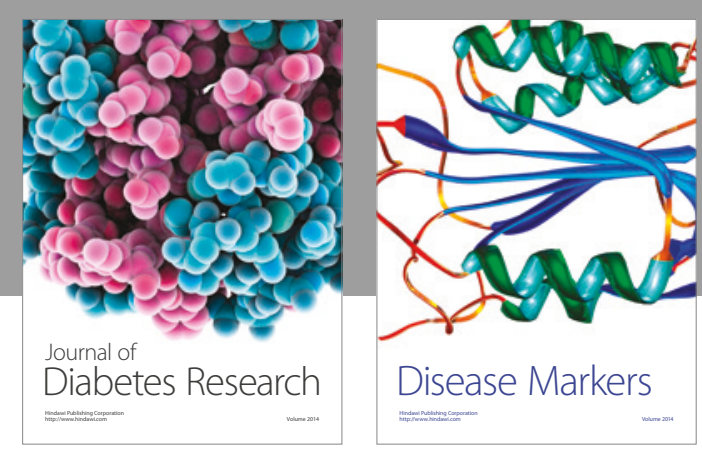

Disease Markers
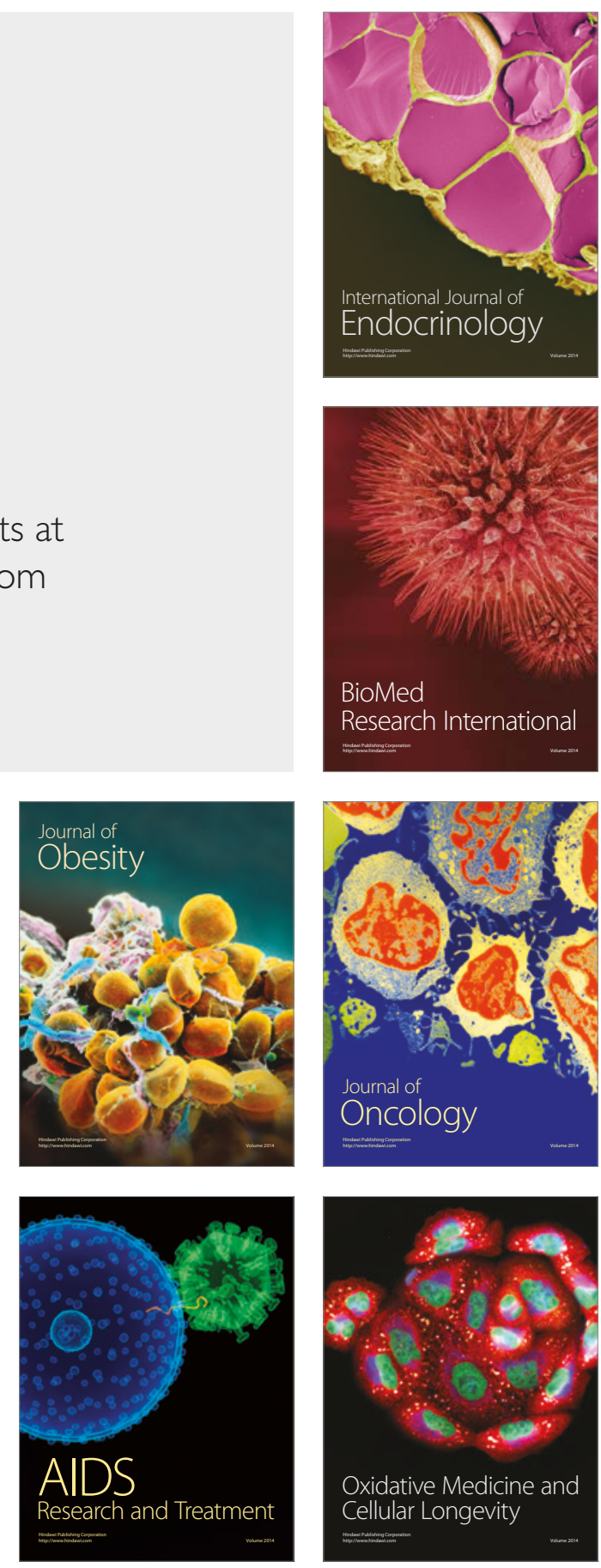